\title{
Double défi pour une communauté francophone
}

La communauté fransaskoise en Saskatchewan

\section{Florent Bilodeau}

\section{CpenEdition}

Journals

Édition électronique

URL : http://journals.openedition.org/ries/3317

DOI : 10.4000/ries.3317

ISSN : 2261-4265

Éditeur

Centre international d'études pédagogiques

Édition imprimée

Date de publication : 1 juin 1996

Pagination : 83-90

ISSN : $1254-4590$

Référence électronique

Florent Bilodeau, "Double défı pour une communauté francophone », Revue internationale d'éducation de Sèvres [En ligne], 10 | 1996, mis en ligne le 30 juillet 2013, consulté le 01 mai 2019. URL : http:// journals.openedition.org/ries/3317 ; DOI : 10.4000/ries.3317

Ce document a été généré automatiquement le 1 mai 2019.

(c) Tous droits réservés 


\title{
Double défi pour une communauté francophone
}

\author{
La communauté fransaskoise en Saskatchewan
}

\author{
Florent Bilodeau
}

1 Tenter d'expliquer à un lectorat international la réalité des nouvelles écoles françaises de la composante fransaskoise en Saskatchewan, en plein centre des prairies canadiennes, voilà un défi qui fait réfléchir et hésiter. Étant situées en milieu agricole, il s'agit de petites écoles presque toutes en milieu rural. Pour comprendre leur situation, il convient d'abord de la situer dans le contexte général du fonctionnement et du rôle des écoles rurales en Saskatchewan, puis de dresser un profil de la nouvelle composante fransaskoise au sein du système scolaire, avec les particularités de sa pédagogie, de ses structures, de ses moyens et de son rôle social, culturel et économique. Cette analyse conduira, en dernier lieu, à présenter les défis que doivent relever les écoles fransaskoises au début du deuxième siècle d'éducation française en terre saskatchewanaise.

2 En 1996, la Saskatchewan compte 194562 élèves de la maternelle à la douzième année (cinq ans à dix-sept/dix-huit ans). De ces élèves, 92374 sont inscrits dans les écoles rurales publiques, séparées (catholiques) ou fransaskoises. Fait à remarquer, des sept écoles secondaires indépendantes (regroupant trois mille élèves), cinq sont situées en milieu rural ou urbain, elles sont gérées par des conseils scolaires, Boards of Education (dix urbains et cent dix ruraux) élus par les contribuables des régions géographiques établies par une loi provinciale. Depuis près de dix ans, les études sur la nécessité de réduire le nombre de divisions scolaires, vu la décroissance des inscriptions ainsi que les nouveautés en communication et en transport, se multiplient. Au moment de la rédaction de cet article, le gouvernement de la province encourage subtilement l'amalgamation des divisions scolaires dans le but d'en réduire considérablement le nombre (de moitié, voire même des deux tiers). La motivation de cette amalgamation tient de l'efficacité administrative, de l'efficacité économique et de la volonté d'affecter le plus de ressources possible à la salle de classe. 
Les écoles rurales en Saskatchewan se présentent sous différentes formes. On y trouve des écoles regroupant la maternelle à la quatrième année (cinq à neuf ans) ou la maternelle à la huitième année (cinq à treize ans) ou la maternelle à la douzième année (cinq à dix-sept ans) ou l'intermédiaire seulement (onze à treize ans) ou encore, le secondaire seulement (quatorze à dix-sept ans). Le choix d'option des niveaux offerts appartient à chaque conseil scolaire. Dans certaines régions plus isolées, ces écoles regroupent une vingtaine d'élèves seulement tandis que des régions où la population rurale est plus concentrée, nous trouvons des écoles de cinq cents à six cents élèves. Indépendamment de l'option offerte, la programmation de chaque niveau scolaire est en grande partie la même puisqu'elle est dérivée du programme cadre défini par le ministère de l'Éducation. Bien sûr, dans les plus petites écoles, nous retrouvons des classes multi-âges sous la responsabilité d'un seul enseignant. Voyant venir l'obligation de regrouper ces petites écoles dans un avenir rapproché, les divisions scolaires n'ont pas osé investir dans l'amélioration des installations matérielles. Conséquemment, plusieurs petites écoles ne sont pas équipées de laboratoires de science et d'informatique ou d'un gymnase. Pour le moment, l'amélioration de cette situation pourrait être réalisée par deux différentes approches : par le transport des élèves vers une installation plus grande et mieux équipée ou encore en reliant le personnel et les élèves de plusieurs écoles par l'informatique ou la télévision interactive. La nouvelle la plus rassurante pour les écoles rurales de la Saskatchewan est l'engagement du gouvernement provincial à assurer la qualité de l'instruction publique dans les milieux ruraux. C'est l'engagement répété par le ministre de l'Éducation lors de sa présentation du budget provincial le 28 mars 1996. Pour le ministre, l'amalgamation des divisions scolaires en milieu rural n'est pas synonyme d'un plus petit nombre d'écoles; il s'agit plutôt d'une volonté d'utiliser les crédits financiers plus efficacement.

Inscriptions dans les écoles fransaskoises au 30 septembre 1995

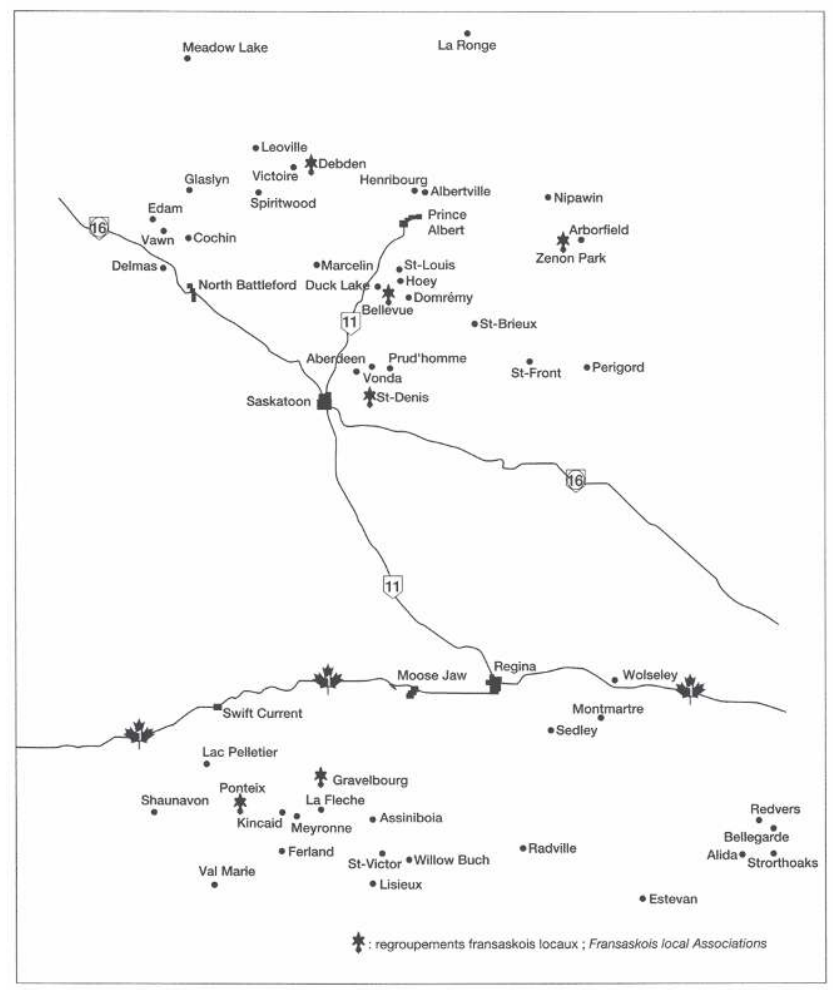




\section{Historique de l'éducation française en Saskatchewan}

L'éducation française existe en Saskatchewan depuis l'arrivée des pionniers (vers 1860) qui venaient défricher les terres arables des prairies canadiennes. Parmi ceux-ci, se trouvaient bon nombre de Canadiens français originaires du Québec, du nord-est des États-Unis, ou encore des pays francophones de l'Europe. Souvent, ces nouveaux fermiers se regroupaient selon leur langue et leur lieu d'origine. Il était donc normal que dans les communautés francophones, la langue du commerce et des institutions publiques soit le français: chaque localité étant autosuffisante, il était donc logique que la langue de l'école soit le français. Avec la création d'un gouvernement provincial en 1905 et la vague d'immigration des pays de l'Europe de l'Est après la Première Guerre mondiale, les contextes démographique et sociologique ont changé radicalement et les communautés francophones ont vu leur pourcentage de population diminuer ainsi que leur homogénéité communautaire s'affaiblir. Ces bouleversements démographiques, doublés d'un accroissement géométrique de la population, ont conduit à la création, en 1944, des Greater School Units (la régionalisation des autorités scolaires) dans lesquelles se sont vues engloutir les communautés canadiennes françaises. Voulu ou non, ce fut un coup mortel porté à l'éducation française en Saskatchewan qui est venu accélérer les efforts des leaders qui s'évertuaient à faire de la Saskatchewan une province unilingue anglaise. Cette perte presque totale de la gestion des écoles homogènes françaises par les francophones a entraîné un processus effarant d'assimilation. Bien que les cinquante dernières années aient servi à disséminer le nombre d'écoles où prime la langue française, elles n'ont pas réussi à atteindre la fierté et la ténacité d'un grand nombre de Saskatchewanais d'origine francophone (connus sous le qualificatif de "fransaskois »). Leurs efforts et leurs revendications ont abouti à l'amendement de la loi scolaire le 3 juin 1993 (qui a redonné aux Fransaskois le droit de gérer leurs écoles), à la création de zones francophones d'éducation en avril 1994, à la première élection de conseillers fransaskois le 24 juin 1994 et au transfert de la gestion des écoles fransaskoises aux nouveaux conseils scolaires fransaskois le $1^{\text {er }}$ janvier 1995.

\section{Fonctionnement de l'école en milieu minoritaire}

5 L'application de ce nouveau droit et de ses implications pour les communautés fransaskoises qui mettent en œuvre ses principes, présente un défi de taille. Malgré le nombre restreint d'inscriptions dans les neuf écoles de la composante fransaskoise (huit cent quarante-cinq élèves au total ${ }^{1}$ ), les dotations pour la programmation sont, pour ainsi dire, les mêmes que pour les écoles de la population majoritaire. Pour les quatre premières années d'implantation, il y a aussi un supplément subventionné par un accord avec le gouvernement du Canada. Bien que trois des écoles fransaskoises soient situées dans des centres urbains (Regina, Saskatoon et Prince Albert), elles sont considérées comme des écoles rurales. De plus, les neuf écoles de la composante sont influencées par la distance et l'isolement l'une de l'autre ${ }^{2}$. En effet, quarante-cinq kilomètres s'étendent entre les deux écoles les plus rapprochées (école canadienne française de Saskatoon et l'école Providence à Vonda) tandis que les deux écoles les plus éloignées (l'école de Bellegarde et l'école Père Mercure à North Battleford) sont séparées par six cent quatrevingts kilomètres. Pour assurer et faciliter l'offre et l'appui de services spéciaux, les 
conseils scolaires fransaskois (CSF), responsables de la gestion de ces neuf écoles, sont regroupés au sein du conseil général des écoles fransaskoises (CGEF). Par le biais de ce regroupement, ils visent à offrir des services professionnels dans les domaines de la programmation académique, de la francisation, de l'éducation spécialisée destinée aux élèves doués ou ayant des difficultés d'apprentissage, de l'informatique, de l'éducation à distance et de la communication.

Inscriptions dans les écoles fransakoises au 30 septembre 1995

\begin{tabular}{|c|c|c|}
\hline ÉCOLES & Niveaux & 1995-1996 \\
\hline École canadienne française de Saskatoon...$\ldots \ldots$. & Mat $-11^{\mathrm{e}}$ année & 154 \\
\hline École Providence (Vonda) $\ldots \ldots \ldots \ldots \ldots \ldots \ldots \ldots$ & Mat - $10^{e}$ année & 81 \\
\hline École Père Mercure (North Battleford) ......... & Mat $-8^{e}$ année & 31 \\
\hline École Valois (Prince Albert) $\ldots \ldots \ldots \ldots \ldots \ldots \ldots$ & Mat - $10^{e}$ année & 74 \\
\hline École St Isidore (Bellevue) $\ldots \ldots \ldots \ldots \ldots \ldots \ldots$ & Mat $-12^{\mathrm{e}}$ année & 127 \\
\hline École de Bellegarde $\ldots \ldots \ldots \ldots \ldots \ldots \ldots \ldots \ldots$ & Mat $-12^{\mathrm{e}}$ année & 85 \\
\hline École Monseigneur de Laval $\ldots \ldots \ldots \ldots \ldots \ldots \ldots$ & Mat $-12^{\mathrm{e}}$ année & 247 \\
\hline École Beau Soleil (Gravelbourg) ............. & Mat $-7^{\mathrm{e}}$ année & 30 \\
\hline École de Ferland $\ldots \ldots \ldots \ldots \ldots \ldots \ldots \ldots \ldots \ldots \ldots \ldots \ldots$ & Mat $-8^{\mathrm{e}}$ année & 16 \\
\hline \multicolumn{2}{|c|}{ 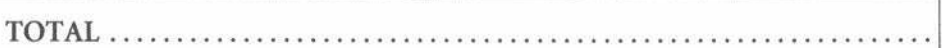 } & 845 \\
\hline
\end{tabular}

\section{Structures et moyens}

Dans la composante fransaskoise comme pour les écoles de la majorité, les installations matérielles varient beaucoup selon le nombre d'élèves et la démographie de la région. Pour les écoles qui accueillent le primaire seulement et dont les inscriptions sont en deçà de cent élèves, on y trouve rarement un gymnase ou des locaux pour l'enseignement des sciences naturelles; pour les établissements incluant le cycle secondaire, ces salles existent là où les inscriptions dépassent soixante-quinze élèves. Dans les deux cas, les écoles sont équipées d'ordinateurs, le nombre de logiciels et didacticiels variant selon les décisions du conseil scolaire. Très peu d'écoles rurales de la majorité sont équipées pour l'enseignement professionnel; aucune des écoles fransaskoises n'a ces équipements. Lorsqu'on dit "rural », on dit agriculture, élevage, foresterie, etc., alors la plupart des élèves sont transportés, grâce à un financement public, sur des distances pouvant aller jusqu'à soixante-quinze, même cent kilomètres.

\section{Programmes et pédagogie}

7 Comme le montrent les chiffres mentionnés plus haut, plus de $98 \%$ des enfants d'âge scolaire en Saskatchewan sont inscrits dans le système public d'éducation, qu'il soit régulier, séparé ou fransaskois. Les services dispensés par ces écoles publiques étant financés par les taxes foncières et les taxes provinciales, elles doivent se conformer aux programmes de base développés par le ministère de l'Éducation. Par conséquent, ce sont les mêmes matières qui sont enseignées aux niveaux primaire, intermédiaire ou secondaire. Les deux différences majeures sont l'enseignement obligatoire de la catéchèse dans les écoles séparées et, dans les écoles de la composante fransaskoise, l'enseignement dispensé en français pour toutes les matières (sauf l'anglais). Fait à préciser, le cours d'anglais dans les écoles fransaskoises est le même que celui enseigné dans les écoles de la 
majorité; il ne s'agit pas d'un cours de langue seconde. Vu la majorité des jeunes Fransaskois se préparant à intégrer un monde du travail où la langue anglaise prime, les parents insistent pour que leurs enfants maîtrisent la langue de la majorité.

Il existe deux différences majeures entre les programmes des écoles rurales et urbaines, et ceci surtout en ce qui concerne les écoles secondaires : la gamme de choix des cours facultatifs et l'offre de cours techniques. L'une et l'autre s'explique par le nombre restreint d'inscriptions qui influe sur les revenus des conseils scolaires et ne permet pas d'offrir le même éventail de cours spécialisés. De plus, les coûts souvent élevés des équipements techniques jouent contre les petites écoles.

Pour ce qui est des approches pédagogiques, elles sont en bonne partie les mêmes en campagne qu'en ville. Les enseignements, encadrés par les programmes du ministère qui précisent les matières obligatoires et les éléments de chaque niveau, doivent aussi les "apprentissages essentiels communs» tels la communication, l'analyse numérique, l'apprentissage autonome, les valeurs personnelles et sociales, l'initiation à la technologie, la créativité et le raisonnement critique. L'instruction est normalement dispensée en situation de classe mettant en interaction un enseignant et un groupe moyen de vingt-deux élèves. Deux changements modifient l'approche de l'enseignement : l'apprentissage par ordinateur qui inclut l'utilisation de réseaux provinciaux, nationaux, voire même d'Internet. En outre, dans les écoles rurales, un facteur qui nécessite une approche pédagogique spéciale est la classe multi-âge. Dans les écoles de la majorité, on y trouve souvent des classes qui regroupent deux années scolaires, même au secondaire. Dans les écoles fransaskoises, la même classe peut offrir trois et même quatre années scolaires. Dans de telles situations, l'apprentissage autonome n'a pas besoin d'être planifié, c'est une conséquence logique. Il est à noter que les recherches éducationnelles démontrent que les élèves qui ont fait leurs études dans ces petites écoles s'adaptent mieux au monde autonome des études universitaires ou collégiales ainsi qu'au monde du travail.

\section{Rôles social, économique et culturel}

10 En plus de dispenser les contenus académiques définis et encadrés par le ministère de l'Éducation, l'école rurale se voit accorder d'autres rôles importants. De plus en plus, c'est elle qui remplace la paroisse ou le silo comme facteur de ralliement social ou économique. Les membres d'une communauté rurale associent la vitalité ou la raison d'être de leur village à l'existence de l'école. Lorsqu'un conseil scolaire envisage la fermeture d'une école qu'il juge non rentable, il n'est pas surprenant que ce conseil soit accusé par les contribuables affectés de causer la disparition éventuelle de leur village. Chaque communauté rurale attend aussi que son école enseigne et fasse la promotion des valeurs qui lui sont chères.

11 Ensuite, l'école rurale, y compris l'école fransaskoise, doit aussi s'efforcer de préparer ses élèves à l'éventualité de vivre en milieu urbain; l'inverse n'existe pas pour l'école urbaine. Puisque dans bien des villages l'école est l'employeur principal, elle représente un élément important dans la vie économique de la communauté. Sa présence ainsi que la perception de sa permanence déterminent les commerçants à s'installer ou à demeurer en activité. 

importance. Ce rôle est aussi important que l'enseignement de la langue elle-même. Les communautés fransaskoises, étant entourées d'un univers anglophone, sont de moins en moins homogènes. Les enfants arrivent à l'école en ayant souvent plus de facilité à s'exprimer en anglais qu'en français. On entend fréquemment dire, qu'en Saskatchewan, le français s'apprend tandis que l'anglais s'attrape. En plus de l'anglicisation du milieu quotidien du jeune, il y a aussi l'influence de la musique et de la télévision américaines. Par conséquent, le personnel des écoles fransaskoises doit ajouter la tâche supplémentaire de franciser bon nombre d'élèves avant de pouvoir leur enseigner les matières obligatoires dans la langue de Molière. Il doit également valoriser l'héritage et la culture des parents en intégrant autant de contenu culturel francophone dans toutes les matières que dans les activités parascolaires.

En Saskatchewan, si les conseils ruraux ne veulent pas succomber à la vague de centralisation qui traverse le pays, ils doivent s'efforcer de bien cerner les valeurs, les attentes et les besoins de leur public scolaire. Ils doivent être de plus en plus novateurs dans leurs approches pour y répondre. Ils ont probablement des leçons à apprendre des petites communautés fransaskoises qui ont su faire valoir leurs points de vue et leurs besoins pour arriver à obtenir la gestion de l'éducation de leurs enfants. Il va sans dire que l'accès aux réseaux électroniques ainsi qu'à la télévision interactive leur permettra de transmettre une éducation de qualité à leurs élèves plutôt que d'être obligés de les transporter vers les écoles des grands centres.

\section{BIBLIOGRAPHIE}

Bashutski B., « Schools of the Future », The School Trustee, 43 (I), 1990, p. 21-23.

Comité de coordination pour le contrôle et la gestion des écoles francophones par les francophones (E. Gallant, président), Une composante du système scolaire de la Saskatchewan au service fransaskois, Saskatchewan, ministère de l'Éducation, juin 1989.

Gajadharsingh J.-L., The Multi-Grade Classroom in Canadian Schools: An Exploratory Study, Department of Curriculum Studies, University of Saskatchewan (Edited Report :The Multi-Grade Classroom: Myth \& Reality-A Canadian Study), CEA Report, 1991.

Gajadharsingh J.-L., Melvin C., «The Multi-Grade Classroom and Student Achievement », The School Trustee, 40 (4), 1987, p. 21-24.

Landry R., « Le bilinguisme additif chez les francophones minoritaires du Canada », Revue des sciences de l'éducation, 8 (1982), p. 223-244.

Langlois H.-D, Scharf M.-P, School Finance and Gouvernance Review: Final Report, Regina SK, School Finance and Governance Review, 1991.

Scharf M.-P., A Report on the Declining Rural Population and the Implications for Rural Education, Regina SK, Saskatchewan School Trustees' Association, Research Centre, Report nº 17, 1974.

Vézina M, « La classe multiprogramme », Dossier Dimensions, avril 1987, p. 13. 


\section{NOTES}

1. Voir tableau infra.

2. Voir supra la carte des communautés fransaskoises.

\section{RÉSUMÉS}

Les neuf écoles de la composante fransaskoise, dispersés sur le territoire de la Saskatchewan, constituent une zone d'éducation francophone. Ces écoles qui doivent appliquer les contenus académiques définis par le ministère de l'Éducation, assurent non seulement un enseignement en français mais jouent également un rôle social, économique et culturel original. Les réseaux électroniques et la télévision interactive seront des outils précieux pour leur réussite.

INDEX

Index géographique : Canada, Saskatchewan

Mots-clés : école rurale, enseignement du français, nouvelles technologies, milieu rural, formation à distance

\section{AUTEUR}

\section{FLORENT BILODEAU}

Directeur général, Conseil général des écoles fransaskoises, Regina, Saskatchewan, Canada. 